Pacific Journal of Mathematics

THE GALOIS CONNECTION BETWEEN PARTIAL FUNCTIONS 


\title{
THE GALOIS CONNECTION BETWEEN PARTIAL FUNCTIONS AND RELATIONS
}

\author{
ISIDORE Fleischer AND Ivo G. ROSENBERG
}

\begin{abstract}
We take up the work of David Geiger in Pacific J. Math., 27 (1968), 95-100 which extends the "abstract" or set theoretic Galois theory from total to partial functions (presumably independently of the previous work dating back to the thirties which is not mentioned). Although Geiger claims to treat even multi-valued functions, it is clear that the basic definition of commutativity he gives on $p$. 96 only makes sense for single-valued partial functions; and although he offers some brief comments towards the end on how one might treat an infinite base set, his results are restricted to finitary functions and relations on a finite set. We develop his characterizations of the Galois-closed classes in a form which is valid for the infinitary case as well. For the classes of partial functions we obtain the infinitarily valid formulation of his criterion; for the relations we find that we must also require closure under an additional operation which we call "duplication", and which is also necessary in the finite case. Indeed, to convince oneself that Geiger's list is incomplete* even for finitary relations on a finite set, one need only observe that the set of finite Cartesian powers is closed in his sense; but the smallest Galois-closed class, i.e., preserved by all partial functions, must surely include the diagonals as well.
\end{abstract}

Since we wish to deal with functions and relations of an arbitrary number, even infinite, of arguments, we find it convenient to index the arguments with an arbitrary set $I$ rather than with a cardinal: an I-ary relation on a set $X$ will thus be a subset of the set of " $I$-tuples", $X^{I}$ (as usual this subset may be described by its characteristic function which, when taken to have values \{true, false\}, may be construed as an $I$-ary "predicate" on $X$ ); an I-ary function on $X$ will be a map from $X^{I}$ to $X$; and an $I$-ary partial function, a map from a subset of $X^{I}$ (i.e., from an $I$-ary relation) its domain $D_{f} \subset X^{I}$ to $X$. One calls $I$ the arity of the relation or function.

Geiger proposes to say that a partial function $f$, say of arity $n$, preserves (or as he puts it, "commutes with") an $I$-ary relation $R$ if it does so "wherever defined": i.e., if its extension $f^{I}$ to $I$-tuples sends $R^{n} \cap D_{f}^{I}$ into $R$ : this means that for every $I \times n$ matrix of elements of $X$ each of whose rows is in $R$ and each of whose columns

* However see Remark at end. 
is in the domain of $f$, the application of $f$ to the columns (indexed by $I$ ) will result in an $I$-tuple of $R$.

The partial functions (of various arities) which preserve a relation (hence also each of a set of relations) surely include the projections of the powers of $X^{n}$ on its factors; they contain, with every function, its restriction to any subset of its domain; and they are closed for composition-this is the operation which assigns to an $m$-ary function $f$ and a family $g_{u}$ of $n$-ary functions indexed by the $\mu \in m$ (it should be recalled that $m$ is an in general infinite set) the $n$-ary function defined on just those $n$-tuples for which each of the $g_{u}$ is defined and yield an $m$-tuple of values for which $f$ is defined, the value of $f$ for this $m$-tuple of values then being the value of the composite function. Conversely, we have the generalization to possibly infinite arities of Geiger's

THEOREM 1. A class $F$ of partial functions on $X$, containing the projections as well as all restrictions and compositions of its members, also contains every n-ary partial function $f$ which preserves every $D_{f}$-ary relation preserved by each of its members.

Proof. Let $R$ be the $D_{f}$-ary relation whose elements are the $n$-ary functions in $F$ with domain $D_{f}$. Since $F$ is closed for composition its members preserve $R$ and therefore so does $f$. But the restrictions to $D_{f}$ of the projections of $X^{n}$ belong to $F$, hence to $R$; and $f$ is defined on this $n$-indexed family of $R^{n}$, hence the result of applying $f$ to it is again in $R$. Thus $f$ belongs to $F$.

From an I-ary relation on $X, R \subset X^{I}$, one can form with the help of a transformation $\sigma: J \rightarrow I$, a $J$-ary relation $R \sigma \subset X^{J}$ : the elements of $R \sigma$ being just those " $J$-tuples" i.e., maps from $J$ to $X$ which one obtains by composing with $\sigma$ all the given " $I$-tuples" i.e., maps from $I$ to $X$, which make up $R$.

On the other hand, from a $J$-ary relation $R \subset X^{J}$ one can form the $I$-ary relation $R \sigma^{-1} \subset X^{I}$ of all those maps from $I$ to $X$ whose composition with $\sigma$ is one of the maps from $J$ to $X$ in the given relation $R$. One may verify that if $R$ is preserved by a partial function (hence also by a set of such), then so is $R \sigma^{-1}\left(f^{I}\right.$ acts on $\left(R \sigma^{-1}\right)^{n}=R^{n}\left(\sigma^{n}\right)^{-1}$ as does $f^{J}$ on $\left.R^{n}\left(\sigma^{n}\right)^{-1} \sigma^{n} \subset R^{n}\right)$. Applying this with $\sigma$ a quotient map, a bijection, and an inclusion, it yields that the relations preserved are closed under identification modulo the kernel of $\sigma$ : i.e., the picking out of those maps in $R$ which are constant on the classes of the kernel of $\sigma$ in $J$ (this is sometimes called "diagonalization") and the construing of their totality as a new relation of arity $J / \operatorname{ker} \sigma$ (generalization of Geiger's operation $R$ ); reindexing which consists in replacing $J$ by a one-one image (which includes 
Geiger's "permutation of variables"); and expansion of a $\sigma(J)$-ary relation to an $I$-ary one by construing the maps from $\sigma(J) \subset I$ as maps from $I$ having dummy arguments on the complement of $\sigma(J)$ (Geiger's operation $A$ for infinite arities). Applied to precomposition, which takes $I$-ary relations $R$ to $J$-ary relations $R \sigma$, these specializations furnish duplication (of the values of each map from $I$ to $X$ ) on the (classes of the) kernel of $\sigma$; reindexing again; and an operation of projection which may destroy preservation by partial (although not by total) functions and will therefore not concern us. Finally, the preserved relations of a fixed arity $I$ are obviously closed under arbitrary intersection (including the void intersection which furnishes the universal relation $X^{I}$ ). Conversely, we have the generalization to possibly infinite arities, as well as the rectification, of Geiger's

THEOREM 2. A class $\mathscr{R}$ of relations closed under indentification, reindexing, expansion, and duplication, and which contains the intersection of every subset of its J-ary relations, also contains every $J$-ary relation $Q$ preserved by every $Q$-ary function preserving each of its members.

Proof. Let $\sigma$ be the "evaluation" map from $J$ to $X^{2}$ which assigns to every element of $J$ the $Q$-tuple of values the maps in $Q$ take on it, and let $R$ be the intersection of the $J$-ary relations in $\mathscr{R}$ which are duplications on the kernel of $\sigma$ and contain $Q$. Every $r: J \rightarrow X$ in $R$ induces a $Q$-ary partial function, say $\bar{r}$, on $\sigma(J)$ as domain (because $R$ is itself a duplication on the kernel of $\sigma$ and so $r$ is constant on its classes); and if $\bar{r}$ failed to preserve some relation in $\mathscr{R}$, then by expanding, duplicating, identifying, and reindexing it, one could construct a $J$-ary relation, which was a duplication on the kernel of $\sigma$ and contained $Q$, which $\bar{r}$ also failed to preserve; and that would contradict the definition of $R$. Thus $\bar{r}$ preserves every relation in $\mathscr{R}$, hence also $Q$. But this means that $r \in Q$, and so $R \subset Q$, whence $Q=R$ belongs to $\mathscr{R}$.

REMARK. The referee suggests that what Geiger intended for the definition of a partial (possibly) multi-valued function $f$ to preserve a relation $R$ was that whenever presented with an $I \times n$ matrix having rows in $R$ and columns in $D_{f}$, each of the $I$-tuples obtained by choosing in all possible ways one from among the values which the function takes on the columns: i.e., the product over $I$ of the value sets which $f$ assigns to the columns as arguments, be in $R$. On this interpretation both of Geiger's theorems become correct as he stated them. Theorem 1 above should be modified for multivalued partial functions by increasing the arity of the preserved 
relations from $D_{f}$ to the grah $G$ of $f$-which is equipotent to $D_{f}$ with each of its elements repeated as often as the multiplicity of the values which $f$ takes on it-and by choosing for the relation $R$ the possible $G$-tuples of values which the $n$-ary functions in $F$ take on the so duplicated elements of $D_{f}$. Then when $f$ preserves $R$ there must be some $G$-tuple in $R$ which takes the full run of $f$ 's values on each of the duplicated elements of its domain-whence the function of $F$ furnishing this $G$-tuple will have (the graph of) $f$ as a restriction (of its graph). Theorem 2 should be modified by deleting all reference to duplications, the relation $R$ in the proof now being simply the intersection of all $J$-ary relations in $\mathscr{R}$ which contain $Q$. Since $\mathscr{R}$ is closed for the $\sigma^{-1}, R$ is preserved by every self-map of $J$ which fixes the elements of $Q$ : thus every $r$ in $R$ may be obtained as a $J$-tuple of values taken on by a multi-valued partial $Q$-ary function which sends $Q$ into $R$ hence as before (but now without duplication) preserves $\mathscr{R}$, and so again $r \in Q$. The multi-valued version of Theorem 1 generalizes the single-valued one; the two versions of Theorem 2 are incomparable.

Added in Proof. Theorem 2 is related to the "Characterization Theorem" of A. A. Iskander, Subalgebra systems of powers of partial universal algebras, this Journal 38 (1971), 457-463 MR 46 \#5212 which restricts itself to relations of a fixed finite arity $J$ preserved by finitary partial functions. This restriction on the functions entails that the $J$-ary relations in the class $\mathscr{R}$ must be taken closed both for intersection and updirected union, whence it suffices to see that $\mathscr{R}$ contains the smallest preserved relation generated by each finite $J$-ary relation $Q$ - and this is just the $R \in \mathscr{R}$ identified in the above proof. Besides reindexing, which is the content of Iskander's (b), the $J$-ary relations in $\mathscr{R}$ should be closed for those combinations of the other operations which do not change arity. Of these, identification followed by duplication comes to intersecting with the diagonal on the common kernel which, in the presence of reindexing and availability of intersection, is covered by his (c); while following identification with expansion comes to his (d) (these are equivalent forms for the "continuity", with respect to the closure of the smallest $J$-ary relation in $\mathscr{R}$, of a retraction in $J$ operating by precomposition on $J$-tuples). Inasmuch as performing the identification after the duplication or expansion gives back the original relation, these suffice: precisely, a set of $J$-ary relations closed for intersection, reindexing and identification followed by expansion and duplication (in any way which restores the suppressed indices) consists of just the $J$-ary relations in the class $\mathscr{R}$ of all duplications of expansions of its identifications - a class closed also for identifications, reindexing and 
THE GALOIS CONNECTION BETWEEN PARTIAL FUNCTIONS AND RELATIONS 97 intersection (of like indexed relations).

Received May 23, 1977.

UNIVERSITY OF MONTREAL

Montreal, Canada 



\section{PACIFIC JOURNAL OF MATHEMATICS}

EDITORS

RICHARD ARENS (Managing Editor)

University of California

Los Angeles, California 90024

C. W. Curtis

University of Oregon

Eugene, OR 97403

C. C. MOORE

University of California

Berkeley, CA 94720
J. DUGUNDJI

Department of Mathematics University of Southern Californı Los Angeles, California 90007

R. Finn and J. Milgram Stanford University Stanford, California 94305

\section{ASSOCIATE EDITORS}

E. F. BECKENBACH

B. H. NEUMANN

F. WOLF

K. YoSHIDA

\section{SUPPORTING INSTITUTIONS}

UNIVERSITY OF BRITISH COLUMBIA
CALIFORNIA INSTITUTE OF TECHNOLOGY
UNIVERSITY OF CALIFORNIA
MONTANA STATE UNIVERSITY
UNIVERSITY OF NEVADA, RENO
NEW MEXICO STATE UNIVERSITY
OREGON STATE UNIVERSITY
UNIVERSITY OF OREGON

UNIVERSITY OF SOUTHERNEALIFORNIA STANFORD UNIVERSITY UNIVERSITY OF HAWAII UNIVERSITY OF TOKYO UNIVERSITY OF UTAH WASHINGTON STATE UNIVERSITY UNIVERSITY OF WASHINGTON 


\section{Pacific Journal of Mathematics \\ Vol. 79 , No. 1 \\ May, 1978}

Teófilo Abuabara, A remark on infinitely nuclearly differentiable

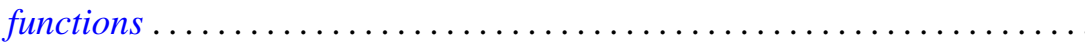

David Fenimore Anderson, Projective modules over subrings of $k[X, Y]$

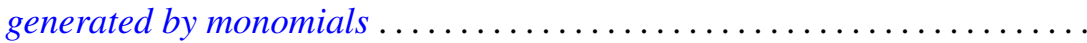

Joseph Barback and Thomas Graham McLaughlin, On the intersection of

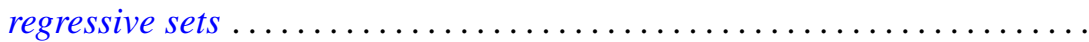

Murray Bell, John Norman Ginsburg and R. Grant Woods, Cardinal inequalities for topological spaces involving the weak Lindelof

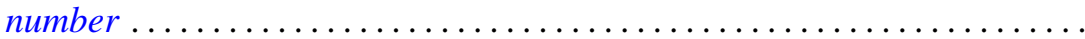

Laurence Richard Boxer, The space of ANRs of a closed surface ............

Zvonko Cerin, Homotopy properties of locally compact spaces at

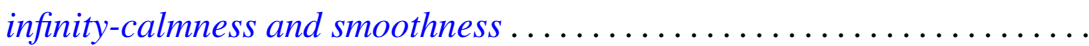

Isidor Fleischer and Ivo G. Rosenberg, The Galois connection between partial functions and relations..................................

John R. Giles, David Allan Gregory and Brailey Sims, Geometrical implications of upper semi-continuity of the duality mapping on a Banach

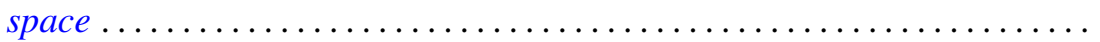

Troy Lee Hicks, Fixed-point theorems in locally convex spaces ............ Hugo Junghenn, Almost periodic functions on semidirect products of transformation semigroups ........................

Victor Kaftal, On the theory of compact operators in von Neumann algebras. II . . . .

Haynes Miller, A spectral sequence for the homology of an infinite

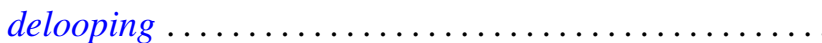

Sanford S. Miller, Petru T. Mocanu and Maxwell O. Reade, Starlike integral operators...

Stanley Stephen Page, Regular FPF rings ...............

Ghan Shyam Pandey, Multipliers for C, 1 summability of Fourier series ...

Shigeo Segawa, Bounded analytic functions on unbounded covering surfaces...

Steven Eugene Shreve, Probability measures and the C-sets of

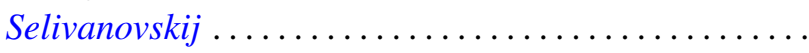

Tor Skjelbred, Combinatorial geometry and actions of compact Lie groups....

Alan Sloan, A note on exponentials of distributions.

Colin Eric Sutherland, Type analysis of the regular representation of a nonunimodular group.

Mark Phillip Thomas, Algebra homomorphisms and the functional

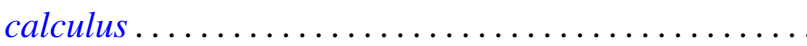

Sergio Eduardo Zarantonello, A representation of $H^{p}$-functions with

$0<p<\infty$. 